

\title{
Singularities of locally acyclic cluster algebras
}

\author{
Angélica Benito, Greg Muller, Jenna Rajchgot and Karen E. Smith
}

We show that locally acyclic cluster algebras have (at worst) canonical singularities. In fact, we prove that locally acyclic cluster algebras of positive characteristic are strongly $F$-regular. In addition, we show that upper cluster algebras are always Frobenius split by a canonically defined splitting, and that they have a free canonical module of rank one. We also give examples to show that not all upper cluster algebras are $F$-regular if the local acyclicity is dropped.

\section{Introduction}

Fomin and Zelevinsky introduced cluster algebras at the close of the twentieth century as a way to study total positivity in a wide range of contexts. Since then, cluster algebra structures have been discovered in many unexpected corners of mathematics (and physics), including Teichmüller theory [Gekhtman et al. 2005; Fock and Goncharov 2007], discrete integrable systems [Fomin and Zelevinsky 2003], knot theory [Muller 2012; Musiker et al. 2013], and mirror symmetry [Shen and Goncharov 2015; Kontsevich and Soibelman 2013], just to name a few.

Locally acyclic cluster algebras, introduced in [Muller 2013], are a large class of cluster algebras which are simultaneously flexible enough to include many interesting examples - including many fundamental examples from representation theory and most examples from Teichmüller theory - yet restrictive enough to avoid the pathological behavior sometimes found in general cluster algebras. For example, locally acyclic cluster algebras are finitely generated and normal, while a general cluster algebra may fail to be either. The main theorem of this paper is that locally acyclic cluster algebras have (at worst) canonical singularities. In fact, we show that locally acyclic cluster algebras of prime characteristic are strongly $F$-regular, a strong form of Frobenius split which implies many nice restrictions on the singularities; for example, $F$-regular varieties are normal, Cohen-Macaulay,

Smith acknowledges the financial support of NSF grant DMS-1001764 and the Clay Foundation. Benito is partially support by MTM2012-35849.

MSC2010: primary 13F60; secondary 13A35, 14B05.

Keywords: cluster algebras, locally acyclic cluster algebras, singularities, $F$-regularity, Frobenius splitting. 
pseudorational, and have Kawamata log terminal singularities (if the canonical class divisor is $\mathbb{Q}$-Cartier) or canonical singularities (if the canonical class is Cartier). These characteristic- $p$ results imply the corresponding statements in characteristic zero as well. For a survey, see, e.g., [Smith 1997b] or [Smith and Zhang 2015].

Associated to a cluster algebra $\mathcal{A}$ is its upper cluster algebra $\mathcal{U}$. This related algebra has the same fraction field and satisfies $\mathcal{A} \subseteq \mathcal{U}$ (see [Berenstein et al. 2005]). We show that all upper cluster algebras in positive characteristic have a "cluster" Frobenius splitting, which can be expressed explicitly in terms of any cluster. We also prove the closely related result that upper cluster algebras have a free canonical module, which is generated by any log volume form in a cluster of cluster variables. The latter of these results is found in the Appendix.

The inclusion $\mathcal{A} \subseteq \mathcal{U}$ need not be equality, though it is in the case when $\mathcal{A}$ is locally acyclic [Muller 2014]. When equality fails, a general philosophy is that $\mathcal{U}$ is better behaved than $\mathcal{A}$. In this direction, we show that if an upper cluster algebra $\mathcal{U}$ fails to be $F$-regular, then $\mathcal{A}$ also fails to be $F$-regular, and we provide an example of this situation. Taking the ground field to be of characteristic zero, this gives an example of a finitely generated upper cluster algebra $\mathcal{U}$ which has $\log$ canonical but not log terminal singularities. We also provide an example where $\mathcal{A} \neq \mathcal{U}$ and $\mathcal{A}$ is pathological (e.g., $\mathcal{A}$ is non-Noetherian), but $\mathcal{U}$ is nevertheless strongly $F$-regular.

All of our results and arguments are also valid for cluster algebras given by an arbitrary skew-symmetrizable matrix. However, we have written the exposition in the slightly less general setting of cluster algebras given by quivers for the sake of accessibility. Experts will have no trouble adapting the arguments to the more general setting.

\section{Cluster algebras}

A cluster algebra is a commutative domain with some extra combinatorial structure. It comes equipped with a (usually infinite) set of generators called cluster variables, which can be recursively generated from a seed: a quiver decorated with a free generating set for a field.

We will consider cluster algebras over an arbitrary field k, although in the literature they are usually defined over $\mathbb{Q}, \mathbb{R}$ or $\mathbb{Z}$. The choice of scalars is mostly irrelevant to the definitions, and most proofs of standard results go through without change. As such, we will cite the original results without comment, and only address the differences as needed.

Seeds and mutations. Let $\mathrm{k}$ be a field, and let $\mathcal{F}$ be a purely transcendental finite extension of $k$. A seed for $\mathcal{F}$ over $k$ consists of the following data:

- A quiver $\mathrm{Q}$ without loops or directed 2-cycles. 

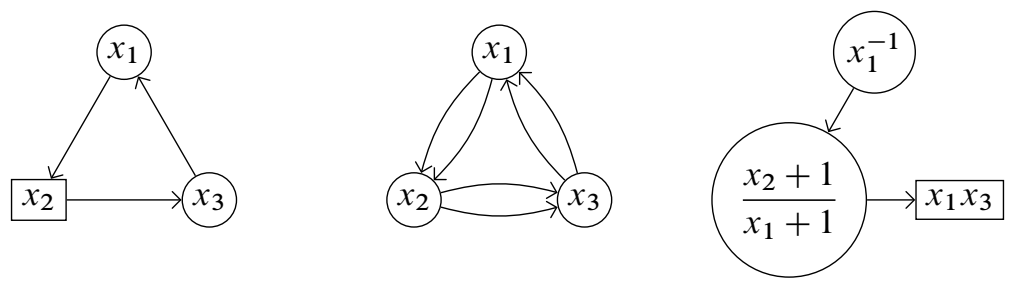

Figure 1. Three examples of seeds in $\mathcal{F}=\mathrm{k}\left(x_{1}, x_{2}, x_{3}\right)$.

- A bijection from the vertices of $Q$ to a set of algebraically independent generators $\boldsymbol{x}=\left\{x_{1}, x_{2}, \ldots, x_{n}\right\}$ for $\mathcal{F}$ over k. The image $x_{i}$ of a vertex $i$ is called the cluster variable at that vertex, and the set $\boldsymbol{x}$ is called a cluster.

- A subset of the vertices of Q designated as frozen; the rest are called mutable. We impose the nonstandard convention that every vertex which touches no arrow is frozen. ${ }^{1}$

Seeds will usually be denoted as a pair $(\mathrm{Q}, \boldsymbol{x})$, with the other data suppressed. The number of vertices of $\mathrm{Q}$ (denoted $n$ hereafter) is the rank of the seed, and the number of mutable vertices (denoted $m$ hereafter) is the mutable rank.

Seeds may be drawn as a quiver with circles $\bigcirc$ for mutable vertices, and rectangles $\square$ for frozen vertices, each with the corresponding cluster variable inscribed (e.g., Figure 1).

A seed $(\mathrm{Q}, \boldsymbol{x})$ may be mutated at any mutable vertex $k$ to produce a new seed $\left(\mu_{k}(\mathrm{Q}), \mu_{k}(\boldsymbol{x})\right)$ for $\mathcal{F}$. The quiver $\mu_{k}(\mathrm{Q})$ is constructed in three steps:

(1) For each pair of arrows $i \rightarrow k \rightarrow j$ through the vertex being mutated, add an arrow $i \rightarrow j$.

(2) Reverse the orientation of every arrow incident to $k$.

(3) Cancel any directed 2-cycles in pairs.

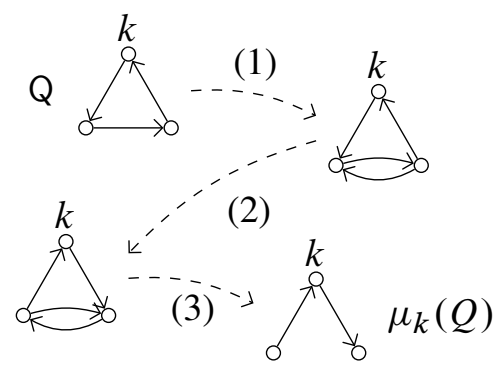

\footnotetext{
${ }^{1}$ This convention allows us to define cluster algebras in characteristic two, and otherwise produces the same definition as the usual convention in every other characteristic. The point is that this convention prevents the numerator in the mutation formula (2-1) from being 2 , which in characteristic two would mean that a mutation at that vertex would not produce another valid cluster variable.
} 
The cluster variables in $\mu_{k}(\boldsymbol{x})$ are the same as those in $\boldsymbol{x}$, except for the cluster variable at vertex $k$, which becomes

$$
x_{k}^{\prime}:=\left(\prod_{i \rightarrow k} x_{i}+\prod_{k \rightarrow i} x_{i}\right) x_{k}^{-1},
$$

where the products are over all arrows into or out of $k$, respectively. Frozen vertices stay frozen.

Mutating at the same vertex twice in a row returns the original seed. That is, mutation is an involution on the set of seeds of $\mathcal{F} / \mathrm{k}$. Two seeds are mutationequivalent if they are related by a sequence of mutations.

Cluster algebras. Fix a seed $(\mathrm{Q}, \boldsymbol{x})$ for $\mathcal{F}$ over $\mathrm{k}$. The union of all the clusters which appear in mutation-equivalent seeds defines the complete set of cluster variables in the ambient field $\mathcal{F}$, naturally grouped into overlapping clusters consisting of those appearing together in a seed. The cluster algebra $\mathcal{A}(\mathrm{Q}, \boldsymbol{x})$ determined by $(\mathrm{Q}, \boldsymbol{x})$ is the sub-k-algebra of $\mathcal{F}$ generated by all of the cluster variables and the inverses of the frozen variables. The cluster algebra only depends on the mutation-equivalence class of the initial seed, and so the initial seed $(Q, x)$ will often be omitted from the notation.

A fundamental property of cluster algebras is the Laurent phenomenon [Fomin and Zelevinsky 2002], which states that each cluster variable can be expressed as a Laurent polynomial in any cluster. Put differently, the localization of $\mathcal{A}$ at any cluster $\boldsymbol{x}=\left\{x_{1}, x_{2}, \ldots, x_{n}\right\}$ is the ring of Laurent polynomials in $\boldsymbol{x}$ over k:

$$
\mathcal{A} \hookrightarrow \mathcal{A}\left[x_{1}^{-1}, x_{2}^{-1}, \ldots, x_{n}^{-1}\right]=\mathrm{k}\left[x_{1}^{ \pm 1}, x_{2}^{ \pm 1}, \ldots, x_{n}^{ \pm 1}\right] \subset \mathcal{F} .
$$

Every cluster in $\mathcal{A}$ defines such an inclusion. This naturally leads to the following definition: the upper cluster algebra $\mathcal{U}$ of $\mathcal{A}$ is the intersection of each of these Laurent rings, taken inside the ambient field $\mathcal{F}$ :

$$
\mathcal{U}:=\bigcap_{\text {clusters } \boldsymbol{x} \subset \mathcal{A}} \mathrm{k}\left[x_{1}^{ \pm 1}, x_{2}^{ \pm 1}, \ldots, x_{n}^{ \pm 1}\right] \subset \mathcal{F} .
$$

By the Laurent phenomenon, there is an inclusion $\mathcal{A} \subseteq \mathcal{U}$. This inclusion is not always equality (see [Berenstein et al. 2005, Proposition 1.26]), but it is an equality in all of the simplest examples, and in many of the most important examples.

Lemma 2.1. Upper cluster algebras are normal.

Proof. Laurent rings over fields are regular, and hence normal. Since an intersection of normal rings inside their common fraction field is normal, upper cluster algebras are normal. 
Cluster localization. Under certain circumstances, localizing a cluster algebra at one or more cluster variables is again a cluster algebra. This important idea was first discussed in [Muller 2013] and further developed in [Muller 2014], to which we refer for more details.

Given a seed $(\mathrm{Q}, \boldsymbol{x})$ over $\mathrm{k}$ and a designated subset $\left\{k_{1}, k_{2}, \ldots, k_{a}\right\}$ of its mutable vertices, we can make a new seed $\left(Q^{\dagger}, \boldsymbol{x}^{\dagger}\right)$ by making those vertices frozen. Because mutations for $\mathrm{Q}^{\dagger}$ are all mutations for $\mathrm{Q}$, there is a natural containment

$$
\mathcal{A}\left(\mathrm{Q}^{\dagger}, \boldsymbol{x}^{\dagger}\right) \subseteq \mathcal{A}(\mathrm{Q}, \boldsymbol{x})\left[x_{k_{1}}^{-1}, x_{k_{2}}^{-1}, \ldots, x_{k_{a}}^{-1}\right] .
$$

If this is an equality, $\mathcal{A}\left(\mathrm{Q}^{\dagger}, \boldsymbol{x}^{\dagger}\right)$ is called a cluster localization of $\mathcal{A}(\mathrm{Q}, \boldsymbol{x})$.

Although it can be difficult to determine whether a particular localization is a cluster localization, there is one situation where it is easy. Indeed, we have inclusions $\mathcal{A}\left(\mathrm{Q}^{\dagger}, \boldsymbol{x}^{\dagger}\right) \subseteq \mathcal{A}(\mathrm{Q}, \boldsymbol{x})\left[x_{k_{1}}^{-1}, x_{k_{2}}^{-1}, \ldots, x_{k_{a}}^{-1}\right] \subseteq \mathcal{U}(\mathrm{Q}, \boldsymbol{x})\left[x_{k_{1}}^{-1}, x_{k_{2}}^{-1}, \ldots, x_{k_{a}}^{-1}\right] \subseteq \mathcal{U}\left(\mathrm{Q}^{\dagger}, \boldsymbol{x}^{\dagger}\right)$,

where the first and third inclusions follow from the fact that the mutations for $\mathrm{Q}^{\dagger}$ are a subset of the mutations for $\mathrm{Q}$ (and the middle inclusion follows from the Laurent phenomenon for $\mathcal{A}(\mathrm{Q}))$. Thus the inclusion in (2-2) is always equality whenever $\mathcal{A}\left(\mathrm{Q}^{\dagger}, \boldsymbol{x}^{\dagger}\right)=\mathcal{U}\left(\mathrm{Q}^{\dagger}, \boldsymbol{x}^{\dagger}\right)$. One extreme case is where we freeze all vertices: since obviously $\mathcal{A}=\mathcal{U}$ when no mutations can happen, it follows that localizing at any full cluster $\boldsymbol{x}$ is a cluster localization. More generally, $\mathcal{A}\left(\mathrm{Q}^{\dagger}, \boldsymbol{x}^{\dagger}\right)=\mathcal{U}\left(\mathrm{Q}^{\dagger}, \boldsymbol{x}^{\dagger}\right)$ will necessarily hold if "enough" mutable vertices become frozen.

For example, if we freeze enough variables to break any directed cycles in $Q$, we arrive at an acyclic quiver $\mathrm{Q}^{\dagger}$. By definition, a quiver is acyclic if it has no directed cycles through mutable vertices; a cluster algebra is acyclic if it admits some acyclic seed. Because acyclic cluster algebras are known to equal their upper cluster algebras (by Theorem 2.6 below), the chain of inclusions (2-3) above implies that $\mathcal{A}\left(\mathrm{Q}^{\dagger}, \boldsymbol{x}^{\dagger}\right)$ is a cluster localization whenever $\mathrm{Q}^{\dagger}$ is acyclic.

Cluster covers. The idea of cluster localization is powerful when a cluster algebra can be covered by cluster localizations.

Definition 2.2. For a cluster algebra $\mathcal{A}$, a set $\left\{\mathcal{A}_{i}\right\}_{i \in I}$ of cluster localizations of $\mathcal{A}$ is called a cluster cover if the corresponding open subschemes cover $\operatorname{Spec}(\mathcal{A})$, that is, if

$$
\operatorname{Spec}(\mathcal{A})=\bigcup_{i \in I} \operatorname{Spec}\left(\mathcal{A}_{i}\right)
$$

If a cluster algebra admits a cluster cover, any geometric property, such as normality, smoothness, or even different classes of singularities, can be checked locally on the cluster localizations. Another property which may be checked on a cover is whether $\mathcal{A}=\mathcal{U}$ : 
Lemma 2.3 [Muller 2014, Lemma 3.3.2]. If $\left\{\mathcal{A}_{i}\right\}_{i \in I}$ is a cluster cover of $\mathcal{A}$, and $\mathcal{A}_{i}=\mathcal{U}_{i}$ for each $i \in I$, then $\mathcal{A}=\mathcal{U}$.

A powerful observation proposed in [Muller 2013] is that many notable classes of cluster algebras admit covers by acyclic cluster algebras.

Definition 2.4. A cluster algebra is locally acyclic if it admits a cluster cover by acyclic cluster algebras.

The class of locally acyclic cluster algebras is much wider than the class of acyclic cluster algebras. The latter class is well-understood and very nicely behaved, but far too restrictive to be itself a major class. On the other hand, locally acyclic cluster algebras include, for example, cluster algebras of Grassmannians, cluster algebras of marked surfaces with at least two marked points on the boundary [Muller 2013, Theorem 10.6], as well as cluster algebras of double Bruhat cells and more generally, positroid cells [Muller and Speyer 2014]. Because the geometric properties of locally acyclic cluster algebras follow nicely from the acyclic case, there is now substantial interest in identifying locally acyclic cluster algebras.

Proposition 2.5 [Muller 2013]. A locally acyclic cluster algebra over $\mathrm{k}$ is finitely generated over $\mathrm{k}$ and equal to its upper cluster algebra. A locally acyclic cluster algebra is normal and a local complete intersection (hence Gorenstein).

This follows with little fuss from the acyclic case, due to Berenstein, Fomin, and Zelevinsky. ${ }^{2}$

Theorem 2.6 [Berenstein et al. 2005, Corollary 1.17; Muller 2014, Corollary 4.2.2]. Let $(\mathrm{Q}, \boldsymbol{x})$ be an acyclic seed. Then the cluster algebra $\mathcal{A}(\mathrm{Q})$ is a finitely generated complete intersection, equal to its upper cluster algebra $\mathcal{U}(\mathrm{Q})$.

Remark 2.7. It is important to note that not every cluster algebra admits a cover by proper cluster localizations. For example, the Markov cluster algebra generated from the middle seed in Figure 1 cannot by covered by proper cluster localizations. Indeed, one easily checks that $\mathcal{A}$ can be $\mathbb{N}$-graded, with every cluster variable homogeneous of degree one. So, any nontrivial cluster localization $\operatorname{Spec}\left(\mathcal{A}_{i}\right)$ of $\operatorname{Spec}(\mathcal{A})$ necessary misses the unique homogeneous maximal ideal generated by the cluster variables.

\section{Frobenius splittings}

Frobenius splittings. Every domain ${ }^{3} R$ over a field of positive characteristic $p$ has a canonical ring map, the Frobenius endomorphism

$$
F: R \rightarrow R, \quad \text { defined by } x \mapsto x^{p} .
$$

\footnotetext{
${ }^{2}$ The proof in [Berenstein et al. 2005] assumes an additional condition, that the cluster algebra is totally coprime. However, it was shown in [Muller 2014] that this condition is unnecessary.

${ }^{3}$ The assumption that $R$ is a domain is completely unnecessary, but it simplifies our discussion and is sufficient for our purposes.
} 
The Frobenius map is an $R$-module map if we equip the target copy of $R$ with the $R$-module structure it gets via restriction of scalars. In practice, it is convenient to denote the target copy of $R$ by some other notation. We denote the target copy by $R^{1 / p}$ and its elements by $r^{1 / p}$, which is consistent with viewing the target copy of $R$ as (the canonically isomorphic ring) $R^{1 / p}$ inside the algebraic closure of the fraction field of $R$. In this case, the elements of $r$ act on elements $x^{1 / p} \in R^{1 / p}$ by $r \cdot x^{1 / p}=\left(r^{p} x\right)^{1 / p}$, the usual multiplication $r x^{1 / p}$ in the fraction field. In this notation, the Frobenius map becomes the inclusion

$$
\begin{aligned}
& R \stackrel{F}{\longleftrightarrow} R^{1 / p}, \\
& r \mapsto\left(r^{p}\right)^{1 / p}=r,
\end{aligned}
$$

of $R$ into the overring $R^{1 / p}$ of $p$-th roots.

We say that $R$ is $F$-finite if $R^{1 / p}$ is a finitely generated $R$-module. This is a fairly weak condition, satisfied, for example, by every finitely generated algebra over a perfect field $k$.

A famous theorem of Kunz [1969, Theorem 2.1] states that an $F$-finite domain $R$ is regular if and only if $R^{1 / p}$ is locally free over $R$. More generally, one should expect that the closer $R^{1 / p}$ is to being locally free over $R$, the milder the singularities of $R$. Frobenius split rings and strongly $F$-regular rings are examples of rings in which some degree of "freeness" is retained of $R^{1 / p}$ over $R$.

Definition 3.1. A domain $R$ is Frobenius split if the map (3-1) splits in the category of $R$-modules. A choice of splitting $\phi: R^{1 / p} \rightarrow R$ is called a Frobenius splitting.

Example 3.2. Every field $k$ of characteristic $p$ is Frobenius split, since $k^{1 / p}$ is a vector space over the subfield k. For a perfect field k, the Frobenius endomorphism is a field isomorphism, and its inverse is the unique Frobenius splitting of $k$.

Example 3.3. Polynomial rings are Frobenius split. Define the standard splitting of the polynomial ring $\mathrm{k}\left[x_{1}, x_{2}, \ldots, x_{n}\right]$ to be given by

$\phi:\left(\mathrm{k}\left[x_{1}, x_{2}, \ldots, x_{n}\right]\right)^{1 / p} \rightarrow \mathrm{k}\left[x_{1}, x_{2}, \ldots, x_{n}\right]$,
$\phi\left(\left(\lambda x_{1}^{a_{1}} x_{2}^{a_{2}} \cdots x_{n}^{a_{n}}\right)^{1 / p}\right)= \begin{cases}\phi\left(\lambda^{1 / p}\right) x_{1}^{a_{1} / p} x_{2}^{a_{2} / p} \cdots x_{n}^{a_{n} / p} & \text { if } a_{1}, a_{2}, \ldots, a_{n} \in p \mathbb{Z}, \\ 0 & \text { otherwise, }\end{cases}$

where the map $\phi: \mathrm{k}^{1 / p} \rightarrow \mathrm{k}$ on scalars $\lambda$ is taken to be any fixed splitting of Frobenius.

Remark 3.4. The standard splitting of a polynomial ring is a Frobenius splitting, and will be the source of Frobenius splittings of cluster algebras. It depends on a choice of generators $\boldsymbol{x}$ and if $\mathrm{k}$ is not perfect, it depends on a choice of Frobenius splitting for $k$. We suppress the dependence on the choice of a Frobenius splitting of $k$ by assuming our ground field comes with a fixed Frobenius splitting. In any case, when $k$ is perfect, there is a unique splitting. 
The standard splitting of a polynomial ring induces a splitting, also called the standard splitting, of the $\mathrm{k}$-Laurent ring $L=\mathrm{k}\left[x_{1}^{ \pm 1}, \ldots, x_{n}^{ \pm 1}\right]$, using the exact same formula as above. An isomorphism between two k-Laurent rings will commute with the standard splitting, so it does not depend on a choice of presentation.

The standard splitting of a k-Laurent ring has the following key property.

Lemma 3.5. If $L$ is a finitely generated Laurent ring over a perfect ${ }^{4}$ field $\mathrm{k}$ of characteristic $p$, then the standard splitting $\phi$ freely generates $\operatorname{Hom}_{L}\left(L^{1 / p}, L\right)$ as an $L^{1 / p}$-module.

Explicitly, every $L$-module map $L^{1 / p} \rightarrow L$ (including every Frobenius splitting) can be written as the composition

$$
L^{1 / p} \stackrel{m_{s}}{\longrightarrow} L^{1 / p} \stackrel{\phi}{\longrightarrow} L
$$

of the standard splitting $\phi$ and "multiplication by $s^{1 / p}$ " map $m_{s}$ for some unique $s \in L$. We denote this composition by $\phi \circ s^{1 / p}$. We include a short proof, although it may be well-known to experts.

Proof. Let $L=\mathrm{k}\left[x_{1}^{ \pm 1}, x_{2}^{ \pm 1}, \ldots, x_{n}^{ \pm 1}\right]$. As an $L$-module, $L^{1 / p}$ has a basis consisting of monomials $\boldsymbol{x}^{\boldsymbol{a}}=x_{1}^{a_{1}} x_{2}^{a_{2}} \cdots x_{n}^{a_{n}}$ for which $0 \leq a_{i}<p$. For any $\psi \in \operatorname{Hom}_{L}\left(L^{1 / p}, L\right)$, define

$$
s:=\sum_{\boldsymbol{a} \mid 0 \leq a_{i}<p} \psi\left(\boldsymbol{x}^{\boldsymbol{a}}\right)^{p} \boldsymbol{x}^{-\boldsymbol{a}} .
$$

Then, for any $\boldsymbol{b}$ with $0 \leq b_{i}<p$,

$$
\phi\left(\left(s x^{b}\right)^{1 / p}\right)=\phi\left(\left(\sum \psi\left(x^{a}\right)^{p} x^{b-a}\right)^{1 / p}\right)=\sum \psi\left(x^{a}\right) \phi\left(x^{b-a}\right)^{1 / p}=\psi\left(x^{b}\right) .
$$

Since $\phi \circ s^{1 / p}$ and $\psi$ agree on a basis for $L^{1 / p}$, they coincide.

Remark 3.6. In fact, for any local or graded Gorenstein $F$-finite ring $S$ of characteristic $p$, the module $\operatorname{Hom}_{S}\left(S^{1 / p}, S\right)$ is a free rank-one $S^{1 / p}$-module, since in this case, $\operatorname{Hom}_{S}\left(S^{1 / p}, S\right)$ is a canonical module for $S^{1 / p} .5$ The point of Lemma 3.5 is that for a Laurent ring $L$, a Frobenius splitting gives a natural generator for $\operatorname{Hom}_{L}\left(L^{1 / p}, L\right)$, and hence for the canonical module of $L$.

One special case is for a field. If $\mathcal{F}$ is a field, then $\operatorname{Hom}_{\mathcal{F}}\left(\mathcal{F}^{1 / p}, \mathcal{F}\right)$ is a onedimensional vector space over $\mathcal{F}^{1 / p}$, so we can take any nonzero mapping to be a basis. In particular, if we fix a splitting $\phi: \mathcal{F}^{1 / p} \rightarrow \mathcal{F}$, then every $\psi: \mathcal{F}^{1 / p} \rightarrow \mathcal{F}$ is the composition $\psi=\phi \circ s^{1 / p}$ for some unique $s \in \mathcal{F}$.

\footnotetext{
${ }^{4}$ Perfect is not necessary here, but it suffices for our purposes and simplifies the discussion.

${ }^{5}$ This is a special case of the general "upper shriek" formula for a finite extension $R \rightarrow S$ that $\omega_{S} \cong \operatorname{Hom}_{R}\left(S, \omega_{R}\right)$ [Hartshorne 1966; Bruns and Herzog 1993, Theorem 3.3.7b]. Note that is $S$ if Gorenstein, then so is the isomorphic ring $S^{1 / p}$; hence $\omega_{S} \cong S$.
} 
Frobenius splittings of upper cluster algebras. As we now prove, upper cluster algebras are always Frobenius split. Indeed, there is a natural cluster splitting which is compatible with the cluster structure:

Theorem 3.7. Suppose that $\mathcal{U}$ is an upper cluster algebra over a field $\mathrm{k}$ of positive characteristic. For any cluster $\boldsymbol{x}=\left\{x_{1}, x_{2}, \ldots, x_{n}\right\}$, the standard splitting of $\mathrm{k}\left[x_{1}^{ \pm 1}, x_{2}^{ \pm 1}, \ldots, x_{n}^{ \pm 1}\right]$ restricts to a splitting of $\mathcal{U}$. This splitting of $\mathcal{U}$ does not depend on the choice of cluster.

The point of the proof is the following simple but crucial observation: a subalgebra $R$ of a Frobenius split algebra $S$ is Frobenius split if $\phi\left(R^{1 / p}\right) \subseteq R$, where $\phi$ is some Frobenius splitting for $S$.

Proof. Let $\boldsymbol{x}^{\prime}=\left\{x_{1}^{\prime}, x_{2}, \ldots, x_{n}\right\}$ be the mutation of $\boldsymbol{x}$ at 1 , and let $P_{1}=x_{1} x_{1}^{\prime}$ be the numerator of the mutation (see (2-1)). The standard splitting $\phi_{x}$ of the Laurent ring $L_{\boldsymbol{x}}$ extends to a splitting of the fraction field $\mathcal{F}$ by localization; we check that this splitting restricts to the standard splitting $\phi_{\boldsymbol{x}^{\prime}}$ on the Laurent ring $L_{\boldsymbol{x}^{\prime}}$.

$$
\phi_{\boldsymbol{x}}\left(\left(\boldsymbol{x}^{\prime \alpha}\right)^{1 / p}\right)=\phi_{\boldsymbol{x}}\left(\left(x_{1}^{\prime \alpha_{1}} x_{2}^{\alpha_{2}} \cdots x_{n}^{\alpha_{n}}\right)^{1 / p}\right)=\phi_{\boldsymbol{x}}\left(\left(P_{1}^{\alpha_{1}} x_{1}^{-\alpha_{1}} x_{2}^{\alpha_{2}} \cdots x_{n}^{\alpha_{n}}\right)^{1 / p}\right) .
$$

Since $P_{1}$ does not contain $x_{1}$, the expression inside $\phi_{x}$ is $x_{1}^{-\alpha_{1}}$ times a rational function of $x_{2}, \ldots, x_{n}$. It follows that this is zero, unless $\alpha_{1}=p \beta_{1}$ for some $\beta_{1} \in \mathbb{Z}$. In this case, $\phi_{\boldsymbol{x}}\left(\left(\boldsymbol{x}^{\prime \alpha}\right)^{1 / p}\right)=\phi_{\boldsymbol{x}}\left(\left(x_{1}^{\prime \beta_{1} p} x_{2}^{\alpha_{2}} \cdots x_{n}^{\alpha_{n}}\right)^{1 / p}\right)=x_{1}^{\prime \beta_{1}} \phi_{\boldsymbol{x}}\left(\left(x_{2}^{\alpha_{2}} \cdots x_{n}^{\alpha_{n}}\right)^{1 / p}\right)$. Since this last expression is a Laurent monomial in $\boldsymbol{x}$, we find that

$$
\phi_{x}\left(\left(x^{\prime \alpha}\right)^{1 / p}\right):= \begin{cases}x^{\prime \beta} & \text { if } \alpha=p \beta, \\ 0 & \text { otherwise, }\end{cases}
$$

and so $\phi_{\boldsymbol{x}}=\phi_{\boldsymbol{x}^{\prime}}$ on $\mathcal{F}$. Iterating this argument, we see that every cluster $\boldsymbol{x}$ gives the same splitting on $\mathcal{F}$. Since this splitting preserves each Laurent $\operatorname{ring} L_{\boldsymbol{x}}$, it preserves their intersection $\mathcal{U}$.

The cluster splitting of $\mathcal{U}$ inherits the key property from Lemma 3.5.

Theorem 3.8. Let $\mathcal{U}$ be an upper cluster algebra over a perfect field $k$. The cluster splitting $\phi$ of $\mathcal{U}$ freely generates $\operatorname{Hom}_{\mathcal{U}}\left(\mathcal{U}^{1 / p}, \mathcal{U}\right)$ as a $\mathcal{U}^{1 / p}$-module.

Proof. Consider a $\mathcal{U}$-module map $\psi: \mathcal{U}^{1 / p} \rightarrow \mathcal{U}$. This map induces, by localization, an $\mathcal{F}$-linear map $\psi: \mathcal{F}^{1 / p} \rightarrow \mathcal{F}$, which we also denote (somewhat abusively) by $\psi$. Since $\operatorname{Hom}_{\mathcal{F}}\left(\mathcal{F}^{1 / p}, \mathcal{F}\right)$ is a one-dimensional vector space over $\mathcal{F}^{1 / p}$ generated by the (localization of the) standard splitting $\phi$, we can write $\psi$ as $\phi \circ s^{1 / p}$ for some unique $s \in \mathcal{F}$. We aim to show that $s \in \mathcal{U}$. This will complete the proof, as every $\psi \in \operatorname{Hom}_{\mathcal{U}}\left(\mathcal{U}^{1 / p}, \mathcal{U}\right)$ will then be the composition of the standard splitting with premultiplication by a unique $s^{1 / p}$ in $\mathcal{U}^{1 / p}$.

To show that $s \in \mathcal{U}$, it suffices to show that $s \in L_{\boldsymbol{x}}$, where $L_{\boldsymbol{x}}$ is the Laurent ring on any cluster $\boldsymbol{x}=\left\{x_{1}, x_{2}, \ldots, x_{n}\right\}$. Note that $L_{\boldsymbol{x}}$ is the localization of $\mathcal{U}$ 
at the cluster variables $\left\{x_{1}, x_{2}, \ldots, x_{n}\right\}$. Thus the map $\psi: \mathcal{U}^{1 / p} \rightarrow \mathcal{U}$ induces an $L_{\boldsymbol{x}}$-module map $\psi_{\boldsymbol{x}}:\left(L_{\boldsymbol{x}}\right)^{1 / p} \rightarrow L_{\boldsymbol{x}}$, which we again call $\psi$. By Lemma 3.5, there is a unique $s_{\boldsymbol{x}} \in L_{\boldsymbol{x}}$ such that $\psi_{\boldsymbol{x}}\left(r^{1 / p}\right)=\phi\left(\left(s_{\boldsymbol{x}} r\right)^{1 / p}\right)$ for all $r \in L_{\boldsymbol{x}}$. But now, localizing further to the fraction field $\mathcal{F}$, this map is of course the same as the map $\phi \circ s^{1 / p}$ from the first paragraph; that is, $\phi \circ s^{1 / p}=\phi \circ s_{\boldsymbol{x}}^{1 / p}$. So by the uniqueness of $s$, we see that $s=s_{\boldsymbol{x}} \in L_{\boldsymbol{x}}$. Since this works for any cluster $\boldsymbol{x}$, it follows that $s \in \mathcal{U}$.

Remark 3.9. The existence of the cluster splitting of $\mathcal{U}$ is closely related to the fact that the canonical module $\omega_{\mathcal{U} / \mathrm{k}}$ is free (see Remark 3.6). This is addressed in the Appendix, which also describes the relation to Frobenius splittings.

\section{4. $F$-regularity of locally acyclic cluster algebras}

Strong $F$-regularity is a strengthened form of Frobenius splitting, first introduced by Hochster and Huneke [1988]. Strongly $F$-regular rings have many nice properties: they are Cohen-Macaulay, normal, and have pseudorational singularities, to name a few. Our main theorem in this section is that locally acyclic cluster algebras are strongly $F$-regular.

Strong $\boldsymbol{F}$-regularity. Fix a domain $R$ of characteristic $p>0$. We continue to assume that $R$ is $F$-finite, meaning that $R^{1 / p}$ is finitely generated over $R$. This is always satisfied for algebras finitely generated over a perfect field.

Strong $F$-regularity will be a splitting condition on iterates of the Frobenius map. For any natural number $e$, let $F^{e}: R \rightarrow R$ denote the $e$-th iterate of Frobenius, so that $F^{e}(r)=r^{p^{e}}$ for all $r \in R$. As in the opening paragraphs of Section 3, it is convenient to replace the target copy of $R$ by the canonically isomorphic ring $R^{1 / p^{e}}$ and view the Frobenius map as the inclusion

$$
R \hookrightarrow R^{1 / p^{e}}
$$

inside the algebraic closure of the fraction field of $R$.

If $R \hookrightarrow R^{1 / p}$ splits, it is easy to see that every iterate $R \hookrightarrow R^{1 / p^{e}}$ splits as well. Indeed, if $\phi: R^{1 / p} \rightarrow R$ is a Frobenius splitting, then there is a naturally induced $R$-module splitting $\phi^{e}: R^{1 / p^{e}} \rightarrow R$ induced by composition

$$
R^{1 / p^{e}} \stackrel{\phi^{1 / p^{e-1}}}{\longrightarrow} R^{1 / p^{e-1}} \longrightarrow \cdots \longrightarrow R^{1 / p} \stackrel{\phi}{\longrightarrow} R .
$$

In particular, upper cluster algebras also have cluster splittings $\phi^{e}$ for the inclusions $\mathcal{U} \hookrightarrow \mathcal{U}^{1 / p^{e}}$, and one easily checks (using the same proof) that $\phi^{e}$ is a generator for $\operatorname{Hom}_{\mathcal{U}}\left(\mathcal{U}^{1 / p^{e}}, \mathcal{U}\right)$ as a $\mathcal{U}^{1 / p^{e}}$-module as in Theorem 3.8.

Definition 4.1. An $F$-finite domain $R$ is strongly $F$-regular if for every nonzero element $x \in R$, there exists $e \in \mathbb{N}$ and $\psi \in \operatorname{Hom}_{R}\left(R^{1 / p^{e}}, R\right)$ such that $\psi\left(x^{1 / p^{e}}\right)=1$. 
Though not apparent from its definition, strong $F$-regularity is a geometric property which restricts how bad singularities can be. The next two well-known theorems are examples of this. See also [Smith and Zhang 2015] for a recent survey of $F$-regularity.

Theorem 4.2 [Hochster and Huneke 1989, Theorem 3.1c]. An F-finite regular ring is strongly $F$-regular.

Theorem 4.3. A Noetherian strongly $F$-regular ring is:

(1) Frobenius split;

(2) Cohen-Macaulay and normal [Hochster and Huneke 1989, Theorem 3.1d];

(3) pseudorational (see [Smith 1997a]);

(4) Kawamata log terminal whenever it is $\mathbb{Q}$-Gorenstein [Hara and Watanabe 2002] (or more generally Kawamata log terminal in the sense of Schwede and Smith's result [2010]: there exists a boundary divisor $\Delta$ such that the pair $(X, \Delta)$ is Kawamata log terminal).

Like most good geometric properties, strong $F$-regularity is a local condition; this is essential for our application to locally acyclic cluster algebras.

Lemma 4.4 [Hochster and Huneke 1989, Theorem 3.1a]. A domain $R$ is strongly $F$-regular if and only if $R_{\mathfrak{p}}$ is strongly $F$-regular, for each prime ideal $\mathfrak{p}$.

In practice, to determine whether or not $R$ is strongly $F$-regular, it often suffices to check the condition in the definition for a single element $x$.

Proposition 4.5 [Hochster and Huneke 1989, Theorem 3.3]. Let $R$ be a Noetherian $F$-finite domain which is Frobenius split. If there is some nonzero $c \in R$ such that

(1) $R_{c}=R\left[c^{-1}\right]$ is strongly $F$-regular, and

(2) there exists $e \in \mathbb{N}$ and $\psi \in \operatorname{Hom}_{R}\left(R^{1 / p^{e}}, R\right)$ such that $\psi\left(c^{1 / p^{e}}\right)=1$,

then $R$ is strongly $F$-regular.

Proof. This is a well-known result lacking a precise reference easy for nonexperts to parse (see [Hochster and Huneke 1989, Theorem 3.1a] or [Smith 2000, Theorem 3.10]), so we include a proof. Take any nonzero $x \in R$. By (1), there exists $\psi \in \operatorname{Hom}_{R_{c}}\left(R_{c}^{1 / p^{f}}, R_{c}\right)$ such that $\psi\left(x^{1 / p^{f}}\right)=1$. Since $\operatorname{Hom}_{R_{c}}\left(R_{c}^{1 / p^{n}}, R_{c}\right)=$ $\operatorname{Hom}_{R}\left(R^{1 / p^{n}}, R\right) \otimes_{R} R_{c}$, we know $\psi=\left(1 / c^{q}\right) \widetilde{\psi}$ for some $\tilde{\psi} \in \operatorname{Hom}_{R}\left(R^{1 / p^{n}}, R\right)$ and some natural number $q$, which without loss of generality can be assumed a power of $p$. So $\widetilde{\psi}\left(x^{1 / p^{n}}\right)=c^{q}$. Now, because $R$ is Frobenius split, a splitting $\phi \in \operatorname{Hom}_{R}\left(R^{1 / q}, R\right)$ will send $\left(c^{q}\right)^{1 / q}$ to $c$. Composing this with the map given in (2) will produce a map sending $x^{1 / q p^{e+n}}$ to 1 . So $R$ is strongly $F$-regular. 
Such an element $c$ is a test element for $R$. These types of test elements were first defined in [Hochster and Huneke 1989]; for a recent survey of test elements in this context, see [Smith and Zhang 2015] (more basic) or [Schwede and Tucker 2012] (more advanced).

$\boldsymbol{F}$-regularity of locally acyclic cluster algebras. We now establish the main result of this section, the $F$-regularity of locally acyclic cluster algebras.

Theorem 4.6. A locally acyclic cluster algebra $\mathcal{A}$ over an $F$-finite field $\mathrm{k}$ of prime characteristic is strongly $F$-regular.

Proof. The assumption on the field ensures that $\mathcal{A}$ is $F$-finite. Strong $F$-regularity is a local condition (see Lemma 4.4), and so it can be checked on an open affine cover. Since locally acyclic cluster algebras admit an open affine cover by acyclic cluster algebras, it suffices to prove the theorem for acyclic cluster algebras.

Fix an acyclic seed $(\mathrm{Q}, \boldsymbol{x})$ for $\mathcal{A}$. We induce on the number of mutable vertices to prove that $\mathcal{A}$ is strongly $F$-regular.

First, suppose there is only one mutable variable; call it $x_{1}$. Then

$$
\mathcal{A}=\mathrm{k}\left[x_{1}, x_{1}^{\prime}, x_{2}^{ \pm 1}, \ldots, x_{n}^{ \pm 1}\right] /\left\langle x_{1} x_{1}^{\prime}-p_{1}^{+}-p_{1}^{-}\right\rangle,
$$

where $p_{1}^{+}$and $p_{1}^{+}$are monomials in $x_{2}, \ldots, x_{n}$ with disjoint supports. This is a localization of the hypersurface algebra

$$
S=\mathrm{k}\left[x_{1}, x_{1}^{\prime}, x_{2}, \ldots, x_{n}\right] /\left\langle x_{1} x_{1}^{\prime}-p_{1}^{+}-p_{1}^{-}\right\rangle .
$$

Since at least one of $p_{1}^{+}$and $p_{1}^{-}$is not $1,{ }^{6}$ the corresponding Jacobian ideal contains a monomial in $x_{2}, \ldots, x_{n}$, and so the Jacobian ideal becomes trivial in the localization to $\mathcal{A}$. Hence, $\mathcal{A}$ is regular, so it strongly $F$-regular by Theorem 4.2.

Assume now by induction that any acyclic quiver with $m-1$ mutable vertices defines a strongly $F$-regular cluster algebra.

Let $(\mathrm{Q}, \boldsymbol{x})$ be an acyclic seed with $m$ mutable vertices. Since $\mathrm{Q}$ is acyclic, we can find a vertex which is mutable and admits no arrows to any other mutable vertex a sink. Label that vertex $x_{1}$, and the remaining mutable vertices $x_{2}, \ldots, x_{r}$. Let $\left(\mathrm{Q}^{\dagger}, \boldsymbol{x}^{\dagger}\right)$ be the same seed but with $x_{1}$ also frozen. Since $\left(\mathrm{Q}^{\dagger}, \boldsymbol{x}^{\dagger}\right)$ is also acyclic, $\mathcal{A}\left(\mathrm{Q}^{\dagger}, \boldsymbol{x}^{\dagger}\right)=\mathcal{U}\left(\mathrm{Q}^{\dagger}, \boldsymbol{x}^{\dagger}\right)$ and so

$$
\mathcal{A}\left(\mathrm{Q}^{\dagger}, \boldsymbol{x}^{\dagger}\right)=\mathcal{A}\left[x_{1}^{-1}\right]
$$

is a cluster localization. The seed $\left(\mathrm{Q}^{\dagger}, \boldsymbol{x}^{\dagger}\right)$ is acyclic with $m-1$ mutable vertices, and so by the inductive hypothesis, $\mathcal{A}\left[x_{1}^{-1}\right]$ is strongly $F$-regular.

Since $\mathcal{A}$ is acyclic, the cluster algebra $\mathcal{A}$ coincides with the upper cluster algebra $\mathcal{U}$ (Theorem 2.6), and so the cluster splitting from Theorem 3.7 is a Frobenius splitting

${ }^{6}$ Due to the assumption that mutable vertices must have at least one incident arrow. 
for $\mathcal{A}$. Hence, by Proposition 4.5, it suffices to check only the test element $c:=x_{1}$ in $\mathcal{A}$. We construct a $\psi$ sending $x_{1}^{1 / p^{e}}$ to 1 directly, using the cluster splitting $\phi$.

Let $p_{1}^{+}$and $p_{1}^{-}$be the monomials appearing in the mutation formula at $x_{1}$, so that $x_{1} x_{1}^{\prime}=p_{1}^{+}+p_{1}^{-}$. Choose $e$ large enough that each exponent appearing in $p_{1}^{+}$or $p_{1}^{-}$is less than $p^{e}$. Since there are no arrows from $x_{1}$ to other mutable vertices, $p_{1}^{-}$is a monomial only in the frozen variables; in particular, it is invertible. Consider the map

$$
\psi=\phi^{e} \circ\left(\frac{x_{1}^{\prime}}{p_{1}^{-}}\right)^{1 / p^{e}},
$$

where $\phi^{e}$ is the cluster splitting of $\mathcal{A} \hookrightarrow \mathcal{A}^{1 / p^{e}}$. Then

$$
\psi\left(x_{1}^{1 / p^{e}}\right)=\phi^{e}\left(\left(\frac{x_{1} x_{1}^{\prime}}{p_{1}^{-}}\right)^{1 / p^{e}}\right)=\phi^{e}\left(\left(\frac{p_{1}^{+}}{p_{1}^{-}}+1\right)^{1 / p^{e}}\right) .
$$

Since $p^{e}$ is greater than any exponent in the Laurent monomial $p_{1}^{+} / p_{1}^{-}$, we know that $\phi^{e}$ kills that term, and so $\psi\left(x_{1}^{1 / p^{e}}\right)=1$. By Proposition 4.5 , this shows that $\mathcal{A}$ is strongly $F$-regular. This completes the inductive step and the proof.

Characteristic zero consequences. So far, our results are for cluster algebras over a field of positive characteristic. By a standard miracle, these results imply similar consequences over fields of characteristic zero.

We first need to check that locally acyclic cluster algebras over $\mathbb{Z}$ behave as expected when tensored with a field $\mathrm{k}$. Let $\mathcal{A}_{\mathbb{Z}}$ denote a cluster algebra over $\mathbb{Z}$. Choosing any seed in $\mathcal{A}_{\mathbb{Z}}$ and replacing the cluster with a cluster over $\mathrm{k}$ determines a cluster algebra $\mathcal{A}_{\mathrm{k}}$ over $\mathrm{k}$, which is well-defined up to canonical isomorphism.

Lemma 4.7. If $\mathcal{A}_{\mathbb{Z}}$ is locally acyclic, then $\mathcal{A}_{\mathrm{k}}$ is locally acyclic and $\mathrm{k} \otimes_{\mathbb{Z}} \mathcal{A}_{\mathbb{Z}} \simeq \mathcal{A}_{\mathrm{k}}$.

Proof. If $\mathcal{A}_{\mathbb{Z}}$ is acyclic, then any acyclic seed of $\mathcal{A}_{\mathbb{Z}}$ corresponds to an acyclic seed of $\mathcal{A}_{\mathrm{k}}$ with the same quiver. The presentations of $\mathcal{A}_{\mathbb{Z}}$ and $\mathcal{A}_{\mathrm{k}}$ from Theorem 2.6 coincide except for the ring of scalars, and so $\mathrm{k} \otimes_{\mathbb{Z}} \mathcal{A}_{\mathbb{Z}} \simeq \mathcal{A}_{\mathrm{k}}$.

If $\mathcal{A}_{\mathbb{Z}}$ is locally acyclic, let $\left\{\left(\mathcal{A}_{i}\right)_{\mathbb{Z}}\right\}_{i \in I}$ be a cover by acyclic cluster algebras. By the previous paragraph, $\mathrm{k} \otimes_{\mathbb{Z}}\left(\mathcal{A}_{i}\right)_{\mathbb{Z}} \simeq\left(\mathcal{A}_{i}\right)_{\mathrm{k}}$ is an acyclic cluster localization of $\mathcal{A}_{\mathrm{k}}$. Since extension of scalars sends covers to covers, $\left\{\left(\mathcal{A}_{i}\right)_{\mathrm{k}}\right\}_{i}$ is a cover of $\mathcal{A}_{\mathrm{k}}$. Since the map $\mathrm{k} \otimes_{\mathbb{Z}} \mathcal{A}_{\mathbb{Z}} \rightarrow \mathcal{A}_{\mathrm{k}}$ is locally an isomorphism, it is an isomorphism.

With this in hand, we may prove one of our main theorems.

Theorem 4.8. A locally acyclic cluster algebra over a field $\mathrm{k}$ of characteristic zero has (at worst) canonical singularities.

Proof. Let $\mathcal{A}$ be a locally acyclic cluster algebra over k, and let $\mathcal{A}_{\mathbb{Z}}$ be the corresponding locally acyclic cluster algebra over $\mathbb{Z}$. By the preceding lemma, for any 
prime $p \in \mathbb{Z}$,

$$
\mathbb{F}_{p} \otimes_{\mathbb{Z}} \mathcal{A}_{\mathbb{Z}} \simeq \mathcal{A}_{\mathbb{F}_{p}}
$$

is locally acyclic. By Theorem 4.6, $\mathcal{A}_{\mathbb{F}_{p}}$ is strongly $F$-regular. On the other hand, $\mathcal{A}$ is Gorenstein by Proposition 2.5. Thus, by [Smith 1997a, Theorem 4.3], $\mathcal{A}_{\mathrm{k}}$ has (at worst) rational singularities, but Gorenstein rational singularities are canonical (see the discussion in [Elkik 1981]).

\section{The nonlocally acyclic setting}

What can be said about cluster algebras and upper cluster algebras which are not locally acyclic? We provide examples which demonstrate that strong $F$-regularity is still possible, but not necessary. We also support the general philosophy that $\mathcal{U}$ should be better-behaved than $\mathcal{A}$ by proving that $F$-regularity of $\mathcal{A}$ implies $F$-regularity of $\mathcal{U}$.

We end this section by showing that related algebras, called the lower bound algebras, are always Frobenius split. We do not know whether or not lower bound algebras are always strongly $F$-regular (they are, at least, always Noetherian complete intersections). Nor do we know whether an arbitrary cluster algebra is Frobenius split in general.

$\boldsymbol{F}$-regularity of $\mathcal{A}$ implies $\boldsymbol{F}$-regularity of $\mathcal{U}$. In this section, we consider a completely arbitrary cluster algebra $\mathcal{A}$ (possibly infinitely generated) over a perfect field.

Lemma 5.1. Fix any integer $e \geq 1$ and let $\varphi \in \operatorname{Hom}_{\mathcal{A}}\left(\mathcal{A}^{1 / p^{e}}, \mathcal{A}\right)$. Then $\varphi$ extends uniquely to a map in $\operatorname{Hom}_{\mathcal{U}}\left(\mathcal{U}^{1 / p^{e}}, \mathcal{U}\right)$.

Proof. The map $\varphi$ extends, by localization, to the Laurent ring generated by the cluster variables in any given cluster. Hence, it preserves $\mathcal{U}$, the common intersection of all of these Laurent rings.

Proposition 5.2. If $\mathcal{A}$ is strongly $F$-regular then $\mathcal{U}$ is strongly $F$-regular.

Proof. Suppose that $c$ is a nonzero element of $\mathcal{U}$. Since $\mathcal{A}$ and $\mathcal{U}$ have the same fraction field, there is an $a \in \mathcal{A}$ for which $a c$ is a nonzero element of $\mathcal{A}$. Because $\mathcal{A}$ is strongly $F$-regular, there is an integer $e \geq 1$ and a map $\varphi \in \operatorname{Hom}_{\mathcal{A}}\left(\mathcal{A}^{1 / p^{e}}, \mathcal{A}\right)$ for which $\varphi\left((a c)^{1 / p^{e}}\right)=1$. By Lemma 5.1, we may extend $\varphi$ uniquely to a map $\tilde{\varphi}: \mathcal{U}^{1 / p^{e}} \rightarrow \mathcal{U}$.

Let $m_{a}: \mathcal{U}^{1 / p^{e}} \rightarrow \mathcal{U}^{1 / p^{e}}$ be the multiplication map given by $m_{a}\left(x^{1 / p^{e}}\right)=$ $a^{1 / p^{e}} x^{1 / p^{e}}$. Then the composition $\tilde{\varphi} \circ m_{a}$ is an element of $\operatorname{Hom}_{\mathcal{U}}\left(\mathcal{U}^{1 / p^{e}}, \mathcal{U}\right)$ which maps $c^{1 / p^{e}}$ to 1 .

In what follows, we focus on the $F$-regularity of upper cluster algebras. 


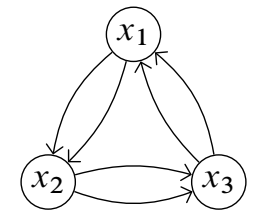

Figure 2. The seed for the Markov cluster algebra in $\mathcal{F}=\mathrm{k}\left(x_{1}, x_{2}, x_{3}\right)$.

The Markov upper cluster algebra. Consider the seed $(\mathrm{Q}, \boldsymbol{x})$ defined in Figure 2. Observe that it has three mutable vertices and no frozen vertices.

Introduced in [Berenstein et al. 2005], the Markov cluster algebra $\mathcal{A}=\mathcal{A}(\mathrm{Q}, \boldsymbol{x})$ is a standard source of counterexamples and pathologies. For example, $\mathcal{A}=\mathcal{A}(\boldsymbol{x}, \mathrm{B})$ is not a locally acyclic cluster algebra, and indeed $\mathcal{A} \subsetneq \mathcal{U}$ [Berenstein et al. 2005, Theorem 1.26]. Moreover, the Markov cluster algebra $\mathcal{A}$ is not Noetherian [Muller 2013].

Nevertheless, the Markov upper cluster algebra $\mathcal{U}=\mathcal{U}(\mathrm{Q}, \boldsymbol{x})$ is quite wellbehaved. It was shown in [Matherne and Muller 2014] that it can be presented as the hypersurface algebra

$$
\mathcal{U} \cong \mathrm{k}\left[x_{1}, x_{2}, x_{3}, M\right] /\left\langle x_{1} x_{2} x_{3} M-x_{1}^{2}-x_{2}^{2}-x_{3}^{2}\right\rangle .
$$

Equivalently, the upper cluster algebra $\mathcal{U}$ is generated inside the field $\mathcal{F}$ by $x_{1}, x_{2}, x_{3}$ and the element

$$
M=\frac{x_{1}^{2}+x_{2}^{2}+x_{3}^{2}}{x_{1} x_{2} x_{3}} .
$$

Proposition 5.3. If $\operatorname{char}(\mathrm{k}) \neq 2,3$, then the Markov upper cluster algebra $\mathcal{U}$ is strongly $F$-regular.

Proof. Since $\mathcal{U}$ is Frobenius split by Theorem 3.7, and the localization of $\mathcal{U}$ at $x_{1} x_{2} x_{3}$ is a Laurent ring, $x_{1} x_{2} x_{3}$ is a test element for $\mathcal{U}$. Consider now the morphism $\varphi: \mathcal{U}^{1 / p^{e}} \rightarrow \mathcal{U}^{1 / p^{e}}$ defined by $\varphi(-)=\phi^{e}\left(\left(1 / 6 \cdot M^{3}\right)^{1 / p^{e}}-\right)$, where $\phi^{e}$ is the iterated cluster splitting of $\mathcal{U}$ defined on page 921 and $M$ is described as before. This morphism $\varphi$ maps $\left(x_{1} x_{2} x_{3}\right)^{1 / p^{e}}$ to 1 :

$$
\begin{aligned}
\varphi\left(\left(x_{1} x_{2} x_{3}\right)^{1 / p^{e}}\right) & =\phi\left(\left(\frac{1}{6}\left(\frac{x_{1}^{2}+x_{2}^{2}+x_{3}^{2}}{x_{1} x_{2} x_{3}}\right)^{3} x_{1} x_{2} x_{3}\right)^{1 / p^{e}}\right) \\
& =\phi\left(\left(\frac{1}{6} \frac{\sum_{i+j \leq 3, i, j \neq 1} c_{i, j} x_{1}^{2 i} x_{2}^{2 j} x_{3}^{6-2 i-2 j}+6 x_{1}^{2} x_{2}^{2} x_{3}^{2}}{\left(x_{1} x_{2} x_{3}\right)^{2}}\right)^{1 / p^{e}}\right) \\
& =1,
\end{aligned}
$$

where the $c_{i, j}$ are some combinatorial coefficients. This shows that $\mathcal{U}$ is strongly $F$-regular. 


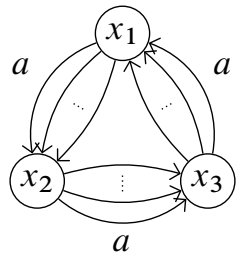

Figure 3. A seed in $\mathcal{F}=\mathrm{k}\left(x_{1}, x_{2}, x_{3}\right)$, where $a \geq 2$.

A non-F -regular upper cluster algebra. Generalizing the previous setting, consider the seed $(\mathrm{Q}, \boldsymbol{x})$ defined in Figure 3 for some integer $a \geq 2$.

Let $\mathcal{U}=\mathcal{U}(\mathrm{Q}, \boldsymbol{x})$ denote the associated upper cluster algebra. As shown in [Matherne and Muller 2014], this generalized Markov upper cluster algebra can be presented as

$$
\mathcal{U} \cong \mathrm{k}\left[x_{1}, x_{2}, x_{3}, M\right] /\left\langle x_{1} x_{2} x_{3} M-x_{1}^{a}-x_{2}^{a}-x_{3}^{a}\right\rangle .
$$

Proposition 5.4. If $a \geq 3$, then $\mathcal{U}$ is not strongly $F$-regular.

Proof. Notice that $\mathcal{U}$ is graded, with

$$
\operatorname{deg}\left(x_{1}\right)=\operatorname{deg}\left(x_{2}\right)=\operatorname{deg}\left(x_{3}\right)=1, \quad \operatorname{deg}(M)=a-3 .
$$

When $a \geq 3$, every homogeneous element in $\mathcal{U}$ has degree at least 0 . As a consequence, the span of the positive degree elements forms a nonzero ideal $I$.

The cluster splitting $\phi$ sends positive degree elements to positive degree elements or zero, so $\phi^{e}\left(I^{1 / p^{e}}\right) \subseteq I$ for any $e$. By Theorem 3.8, any $\psi \in \operatorname{Hom}_{\mathcal{U}}\left(\mathcal{U}^{1 / p^{e}}, \mathcal{U}\right)$ can be written as

$$
\psi=\phi^{e}\left(s^{1 / p^{e}}-\right)
$$

for some $s \in \mathcal{U}$. Since $(s I)^{1 / p^{e}} \subseteq I^{1 / p^{e}}$, we see that $\psi\left(I^{1 / p^{e}}\right) \subseteq I^{1 / p^{e}}$ for any $\psi \in \operatorname{Hom}_{\mathcal{U}}\left(\mathcal{U}^{1 / p^{e}}, \mathcal{U}\right)$. In particular, for any $c \in I$, there is no $\psi \in \operatorname{Hom}_{\mathcal{U}}\left(\mathcal{U}^{1 / p^{e}}, \mathcal{U}\right)$ such that $\psi(c)=1 \notin I$, and so $\mathcal{U}$ is not strongly $F$-regular.

By Proposition 5.2, this extends to the cluster algebra as well.

Corollary 5.5. If $a \geq 3$, then $\mathcal{A}(\mathrm{Q}, \boldsymbol{x})$ is not strongly $F$-regular.

Remark 5.6. The positive degree elements in $\mathcal{U}$ form an ideal $\left(x_{1}, x_{2}, x_{3}\right)$ stable under all maps in $\operatorname{Hom}_{\mathcal{U}}\left(\mathcal{U}^{1 / p^{e}}, \mathcal{U}\right)$, that is, compatible with respect to every element of $\operatorname{Hom}_{\mathcal{U}}\left(\mathcal{U}^{1 / p^{e}}, \mathcal{U}\right)$. This is the test ideal of $\mathcal{U}$. See [Schwede and Tucker 2012].

Lower bound algebras. Fix a seed $(\mathrm{Q}, \boldsymbol{x})$, where $\boldsymbol{x}=\left(x_{1}, x_{2}, \ldots, x_{n}\right)$. As before, let

$$
p_{i}^{+}:=\prod_{j \rightarrow i} x_{j} \quad \text { and } \quad p_{i}^{-}:=\prod_{j \leftarrow i} x_{j}
$$


and let $x_{i}^{\prime}:=\left(p_{i}^{+}+p_{i}^{-}\right) x_{i}^{-1}$. The algebra $\mathcal{L}(\mathrm{Q}, \boldsymbol{x})$ defined by

$$
\mathcal{L}(\mathrm{Q}, \boldsymbol{x}):=\mathrm{k}\left[x_{1}, x_{2}, \ldots, x_{n}, x_{1}^{\prime}, x_{2}^{\prime}, \ldots, x_{n}^{\prime}\right] \subseteq \mathcal{F}=\mathrm{k}\left(x_{1}, \ldots, x_{n}\right)
$$

is called the lower bound algebra associated to the seed $(\mathrm{Q}, \boldsymbol{x})$. Notice that $\mathcal{L}(\mathrm{Q}, \boldsymbol{x}) \subseteq \mathcal{A}(\mathrm{Q}, \boldsymbol{x})$. This inclusion is an equality if and only if $\mathrm{Q}$ is an acyclic quiver (see [Berenstein et al. 2005, Theorem 1.20]).

Lemma 5.7. The kernel L of the surjective ring homomorphism

$$
\mathrm{k}\left[x_{1}, \ldots, x_{n}, y_{1}, \ldots, y_{n}\right] \rightarrow \mathcal{L}(\mathrm{Q}, \boldsymbol{x}), \quad x_{i} \mapsto x_{i}, y_{i} \mapsto x_{i}^{\prime},
$$

is a prime component of the ideal $I:=\left\langle x_{1} y_{1}-\left(p_{1}^{+}+p_{1}^{-}\right), \ldots, x_{n} y_{n}-\left(p_{n}^{+}+p_{n}^{-}\right)\right\rangle$. Proof. Since $\mathcal{L}(\mathrm{Q}, \boldsymbol{x})$ is a domain, $L$ is a prime ideal. To see that $L$ is a component of $I$, let $S=\mathrm{k}\left[x_{1}, \ldots, x_{n}, y_{1}, \ldots, y_{n}\right]$, and observe that

$$
I S\left[\left(x_{1} \cdots x_{n}\right)^{-1}\right]=\left\langle y_{1}-x_{1}^{\prime}, \ldots, y_{n}-x_{n}^{\prime}\right\rangle .
$$

Since $L=\left\langle y_{1}-x_{1}^{\prime}, \ldots, y_{n}-x_{n}^{\prime}\right\rangle \cap S$, it follows that $\left(I S\left[\left(x_{1} \cdots x_{n}\right)^{-1}\right]\right) \cap S=L$, and thus $L$ is a prime component of $I$.

Proposition 5.8. The lower bound algebra $\mathcal{L}(\mathrm{Q}, \boldsymbol{x})$ is Frobenius split.

Proof. Fix any prime $p>0$, let $S:=\mathrm{k}\left[x_{1}, \ldots, x_{n}, y_{1}, \ldots, y_{n}\right]$, and let $\mathcal{B}$ denote the $S$-module basis of $S^{1 / p}$ consisting of all monomials

$$
x_{1}^{a_{1} / p} \cdots x_{n}^{a_{n} / p} y_{1}^{a_{n+1} / p} \cdots y_{n}^{a_{2 n} / p}, \quad 0 \leq a_{i}<p .
$$

Define $\psi: S^{1 / p} \rightarrow S$ to be the $S$-linear map which takes value 1 on the basis element $x_{1}^{(p-1) / p} \cdots x_{n}^{(p-1) / p} y_{1}^{(p-1) / p} \cdots y_{n}^{(p-1) / p}$ and 0 on all other elements of $\mathcal{B}$.

We will construct a Frobenius splitting of $S$ which descends to a Frobenius splitting of $\mathcal{L}$. To this end, let

$$
f=\prod_{1 \leq i \leq n}\left(x_{i} y_{i}-p_{i}^{+}-p_{i}^{-}\right),
$$

and observe that premultiplication of $\psi$ by $f^{(p-1) / p}$ is a Frobenius splitting of $S$. Indeed, since the $p^{+}$and $p^{-}$never involved any $y$, all monomials appearing in $f^{(p-1)}$ involve each $y_{i}$ to a power less than $p$; all of these are killed by $\psi$ except for the monomial term $x_{1}^{(p-1)} \cdots x_{n}^{(p-1)} y_{1}^{(p-1)} \cdots y_{n}^{(p-1)}$, whose $p$-th root gets sent to 1 by $\psi$. So, $\psi\left(f^{(p-1) / p} \cdot 1\right)=1$, and $\psi\left(f^{(p-1) / p_{-}}\right)$is thus a Frobenius splitting of $S$. Furthermore, if $J=\langle f\rangle$, then

$$
\psi\left(f^{(p-1) / p} J^{1 / p}\right) \subseteq J
$$

That is, $J$ is a compatibly split ideal. Because sums and prime components of compatibly split ideals are compatibly split (see, for example, [Brion and Kumar 
2005, Chapter 1.2]), the ideal $L \subseteq S$ that defines the lower bound algebra $\mathcal{L}(\mathrm{Q}, \boldsymbol{x})$ is compatibly split. The Frobenius splitting $\psi\left(f^{(p-1) / p}-\right): S^{1 / p} \rightarrow S$ therefore descends to a Frobenius splitting of the lower bound algebra.

\section{Appendix: The canonical module of an upper cluster algebra}

This appendix considers the canonical module of an upper cluster algebra $\mathcal{U}$ over a field $k{ }^{7}$ Since upper cluster algebras need not be Noetherian [Speyer 2013], we must be careful which definition we use.

Canonical modules. Let $S$ be a normal domain over k such that the fraction field $\mathcal{F}(S)$ has transcendence degree $n$ over k. Define the canonical module of $S$ over k to be the $S$-module

$$
\omega_{S / \mathrm{k}}:=\left(\Lambda_{S}^{n} \Omega_{S / \mathrm{k}}\right)^{* *} .
$$

If $S$ is regular (such as a field), then the double dual in the definition is unnecessary, and $\omega_{S / \mathrm{k}}=\Lambda_{S}^{n} \Omega_{S / \mathrm{k}}$. This construction commutes with localization; in particular, there is a natural embedding

$$
\omega_{S / \mathrm{k}} \subseteq \omega_{\mathcal{F}(S) / \mathrm{k}}=\Lambda_{\mathcal{F}(S)}^{n} \Omega_{\mathcal{F}(S) / \mathrm{k}}
$$

into the canonical module of the fraction field.

The log volume form. Let $\mathcal{U}$ be an upper cluster algebra over $k$. The algebra $\mathcal{U}$ is normal and the transcendence degree of its fraction field over $\mathrm{k}$ is the rank $n$. For a cluster $\boldsymbol{x}$ with functions $\left\{x_{1}, x_{2}, \ldots, x_{n}\right\}$ indexed by $\{1,2, \ldots, n\}$, let $L_{\boldsymbol{x}}$ denote the k-Laurent ring in that cluster, and define the log volume form

$$
\mu_{\boldsymbol{x}}:=\frac{d x_{1} \wedge d x_{2} \wedge \cdots \wedge d x_{n}}{x_{1} x_{2} \cdots x_{n}} \in \omega_{L_{\boldsymbol{x}} / \mathrm{k}}
$$

Note that a permutation of the indices may change the sign of this element.

Proposition A.1. The canonical module $\omega_{L_{x} / k}$ is free of rank one over $L_{\boldsymbol{x}}$ and generated by the log volume form $\mu_{\boldsymbol{x}}$.

The log volume form is an invariant of the cluster algebra, up to sign.

Proposition A.2. For two different clusters $\boldsymbol{x}, \boldsymbol{y}$ of $\mathcal{U}$, we have $\mu_{\boldsymbol{x}}= \pm \mu_{\boldsymbol{y}}$.

Proof. It is sufficient to check the proposition for a single mutation. Let $\boldsymbol{x}^{\prime}=$ $\left\{x_{1}, x_{2}, \ldots, x_{i}^{\prime}, \ldots, x_{n}\right\}$, where $x_{i}^{\prime}=\left(p_{i}^{+}+p_{i}^{-}\right) / x_{i}$. Then

$$
\frac{d x_{i}^{\prime}}{x_{i}^{\prime}}=\frac{d\left(p_{i}^{+}+p_{i}^{-}\right)}{x_{i} x_{i}^{\prime}}-\frac{\left(p_{i}^{+}+p_{i}^{-}\right) d x_{i}}{x_{i}^{2} x_{i}^{\prime}}=\frac{d\left(p_{i}^{+}+p_{i}^{-}\right)}{p_{i}^{+}+p_{i}^{-}}-\frac{d x_{i}}{x_{i}} .
$$

\footnotetext{
${ }^{7}$ The results remain true over $\mathbb{Z}$.
} 
Since $p_{i}^{+}$and $p_{i}^{-}$are monomials in $\left\{x_{1}, x_{2}, \ldots, x_{i-1}, x_{i+1}, \ldots, x_{n}\right\}$,

$$
\frac{d x_{1} \wedge \cdots \wedge d x_{i}^{\prime} \wedge \cdots \wedge d x_{n}}{x_{1} \cdots x_{i}^{\prime} \cdots x_{n}}=-\frac{d x_{1} \wedge \cdots \wedge d x_{i} \wedge \cdots \wedge d x_{n}}{x_{1} \cdots x_{i} \cdots x_{n}} .
$$

Hence, $\mu_{\boldsymbol{x}}=-\mu_{\boldsymbol{x}^{\prime}}$. Iterating mutations or permuting the indices will change this form by at most a sign.

Canonical modules of upper cluster algebras. Since either log volume form freely generates the canonical module after localizing to a cluster Laurent ring, it follows that they freely generate the canonical module of $\mathcal{U}$.

Theorem A.3. For an upper cluster algebra $\mathcal{U}$ over a field ${ }^{8} \mathrm{k}$, the canonical module $\omega_{\mathcal{U} / \mathrm{k}}$ is free of rank one over $\mathcal{U}$ and generated by a log volume form in any cluster. Proof. Fix a $\log$ volume form $\mu$ in some cluster. For any cluster $\boldsymbol{x}$, the localization $L_{\boldsymbol{x}} \otimes \Lambda_{\mathcal{U}}^{n} \Omega_{\mathcal{U} / \mathrm{k}}$ equals $L_{\boldsymbol{x}} \mu$ by Proposition A.1. Let

$$
\Lambda_{\mathcal{U}}^{n} \Omega_{\mathcal{U} / \mathrm{k}} \rightarrow f\left(\Lambda_{\mathcal{U}}^{n} \Omega_{\mathcal{U} / \mathrm{k}}\right)
$$

be the quotient by the maximal torsion submodule. Then $f\left(\Lambda_{\mathcal{U}}^{n} \Omega_{\mathcal{U} / \mathrm{k}}\right)$ is contained inside the localization $L_{\boldsymbol{x}} \mu$, which is contained inside the generic canonical module $\Lambda_{\mathcal{F}(\mathcal{U})}^{n} \Omega_{\mathcal{F}(\mathcal{U}) / \mathrm{k}}$. Intersecting over all clusters, we obtain a map

$$
\Lambda_{\mathcal{U}}^{n} \Omega_{\mathcal{U} / \mathrm{k}} \rightarrow f\left(\Lambda_{\mathcal{U}}^{n} \Omega_{\mathcal{U} / \mathrm{k}}\right) \subseteq \bigcap_{\text {clusters } \boldsymbol{x}}\left(L_{\boldsymbol{x}} \mu\right)=\left(\bigcap_{\text {clusters } \boldsymbol{x}} L_{\boldsymbol{x}}\right) \mu=\mathcal{U} \mu .
$$

Define $\mu^{*} \in\left(\Lambda_{\mathcal{U}}^{n} \Omega_{\mathcal{U} / \mathrm{k}}\right)^{*}=\operatorname{Hom}_{\mathcal{U}}\left(\Lambda_{\mathcal{U}}^{n} \Omega_{\mathcal{U} / \mathrm{k}}, \mathcal{U}\right)$ to be the composition of the above map with the $\mathcal{U}$-module map $\mathcal{U} \mu \rightarrow \mathcal{U}$ which sends $\mu$ to 1 .

Consider another $\mathcal{U}$-module map $\psi: \Lambda_{\mathcal{U}}^{n} \Omega_{\mathcal{U} / \mathrm{k}} \rightarrow \mathcal{U}$. Since $\mathcal{U}$ is torsion-free, $\psi$ factors through $f\left(\Lambda_{\mathcal{U}}^{n} \Omega_{\mathcal{U} / \mathrm{k}}\right)$. Localizing $\psi$ at a cluster $\boldsymbol{x}$ gives an $L_{\boldsymbol{x}}$-module map $\psi_{\boldsymbol{x}}: L_{\boldsymbol{x}} \mu \rightarrow L_{\boldsymbol{x}}$. Let $s_{\boldsymbol{x}}:=\psi_{\boldsymbol{x}}(\mu)$, and note that $\psi_{\boldsymbol{x}}(a \mu)=a s_{\boldsymbol{x}}$ for all $a \in L_{\boldsymbol{x}}$.

Localizing at a different cluster $y$ gives a map $\psi_{\boldsymbol{y}}: L_{\boldsymbol{y}} \mu \rightarrow L_{\boldsymbol{y}}$, which is of the form $\psi_{\boldsymbol{y}}(a \mu)=a s_{\boldsymbol{y}}$ for some $s_{\boldsymbol{y}} \in L_{\boldsymbol{y}}$. Since there is some nonzero $b \in U$ such that $b \mu \in f\left(\Lambda_{\mathcal{U}}^{n} \Omega_{\mathcal{U} / \mathrm{k}}\right)$ (the product of the variables in any cluster suffices),

$$
b s_{\boldsymbol{x}}=b \psi_{\boldsymbol{x}}(\mu)=\psi(b \mu)=b \psi_{\boldsymbol{y}}(\mu)=b s_{\boldsymbol{y}},
$$

and it follows that $s_{\boldsymbol{x}}=s_{\boldsymbol{y}}$ in $L_{\boldsymbol{x}} \cap L_{\boldsymbol{y}}$. Repeating for all clusters, $s_{\boldsymbol{x}} \in \mathcal{U}$. Therefore, $\psi(a \mu)=a s_{\boldsymbol{x}}=\mu^{*}\left(s_{\boldsymbol{x}} a \mu\right)$ for all $a \in \mathcal{U}$; this proves that $\mu^{*}$ freely generates the dual module

$$
\left(\Lambda_{\mathcal{U}}^{n} \Omega_{\mathcal{U} / \mathrm{k}}\right)^{*}=\mathcal{U} \mu^{*}
$$

Dualizing both sides demonstrates that $\omega_{\mathcal{U} / \mathrm{k}}=\mathcal{U} \mu$.

\footnotetext{
${ }^{8}$ The theorem remains true when $\mathrm{k}$ is an arbitrary normal domain.
} 
There are examples where the log volume forms are not in $\Omega_{\mathcal{U} / \mathrm{k}}^{n}$; hence, the double dual in the definition of $\omega_{\mathcal{U} / \mathrm{k}}$ is necessary.

Corollary A.4. A Noetherian upper cluster algebra over a field is Gorenstein.

Canonical modules and Frobenius splittings. We sketch the relation between canonical modules and Frobenius splittings here; further details may be found in [Brion and Kumar 2005, Section 1.3].

Let $\mathrm{k}$ be a field of positive characteristic $p \neq 2$, and let $X$ be a smooth, locally finite-type scheme over $k$. The Frobenius map becomes a flat, finite morphism

$$
f: X \rightarrow X \text {. }
$$

Then the push-forward functor $f_{*}: \operatorname{Coh}(X) \rightarrow \operatorname{Coh}(X)$ has a right-adjoint

$$
f^{!}: \operatorname{Coh}(X) \rightarrow \operatorname{Coh}(X),
$$

together with an adjunction map

$$
\operatorname{tr}: f_{*} f^{!} \rightarrow \text { Id }
$$

called the trace.

The coherent sheaf $f^{!}\left(\mathcal{O}_{X}\right)$ and its trace map can be connected with Frobenius splittings as follows. On any open affine subscheme $\operatorname{Spec}(R) \subseteq X$ :

- $f^{!}\left(\mathcal{O}_{X}\right)[\operatorname{Spec}(R)]$ is isomorphic to $\operatorname{Hom}_{R}\left(R^{1 / p}, R\right)$ as an $R^{1 / p}$-module; equivalently, to $\operatorname{Hom}_{R^{p}}\left(R, R^{p}\right)$ as an $R$-module.

- $f_{*} f^{!}\left(\mathcal{O}_{X}\right)[\operatorname{Spec}(R)]$ is isomorphic to $\operatorname{Hom}_{R}\left(R^{1 / p}, R\right)$ as an $R$-module.

- The trace map is given by the $R$-module map

$$
\operatorname{Hom}_{R}\left(R^{1 / p}, R\right) \rightarrow R,
$$

which sends a map $f: R^{1 / p} \rightarrow R$ to $f(1) \in R$.

Hence, the sheaf of Frobenius splittings is isomorphic to $\operatorname{tr}^{-1}(1) \subset f_{*} f^{!}\left(\mathcal{O}_{X}\right)$.

Duality theory for the morphism $f$ gives an alternate description of $f^{!}\left(\mathcal{O}_{X}\right)$ in terms of the canonical sheaf $\omega_{X / k}$.

Theorem A.5 [Brion and Kumar 2005, §1.3]. There are natural isomorphisms ${ }^{9}$

$$
\begin{aligned}
f^{!}\left(\mathcal{O}_{X}\right) & \stackrel{\sim}{\longrightarrow} \operatorname{Hom}_{X}\left(f^{*} \omega_{X / \mathrm{k}}, \omega_{X / \mathrm{k}}\right) \stackrel{\sim}{\longrightarrow}\left(\omega_{X / \mathrm{k}}\right)^{1-p}, \\
f_{*} f^{!}\left(\mathcal{O}_{X}\right) & \sim \operatorname{Hom}_{X}\left(\omega_{X / \mathrm{k}}, f_{*} \omega_{X / \mathrm{k}}\right) \stackrel{\sim}{\longrightarrow} f_{*}\left(\left(\omega_{X / \mathrm{k}}\right)^{1-p}\right),
\end{aligned}
$$

and a map $\tau: f_{*} \omega_{X / \mathrm{k}} \rightarrow \omega_{X / \mathrm{k}}$, such that the trace map is given by

$$
f_{*} f^{!}\left(\mathcal{O}_{X}\right) \stackrel{\sim}{\longrightarrow} \mathcal{H o m}_{X}\left(\omega_{X / \mathrm{k}}, f_{*} \omega_{X / \mathrm{k}}\right) \stackrel{\tau \circ-}{\longrightarrow} \mathcal{H o m} X\left(\omega_{X / \mathrm{k}}, \omega_{X / \mathrm{k}}\right) \stackrel{\sim}{\longrightarrow} \mathcal{O}_{X} .
$$

\footnotetext{
${ }^{9}$ The negative exponent on $\left(\omega_{X / k}\right)^{1-p}$ denotes a positive power of the dual sheaf $\omega_{X / \mathrm{k}}^{*}$. This is a markedly different use of exponential notation than $R^{1 / p}$.
} 
Back to cluster algebras. Let $\mathcal{U}$ be an upper cluster algebra. For any seed $(\mathrm{Q}, \boldsymbol{x})$, the Laurent phenomenon says that freezing every mutable vertex gives the localization $\mathrm{k}\left[x_{1}^{ \pm 1}, \ldots, x_{n}^{ \pm 1}\right]$ in the cluster $\boldsymbol{x}=\left\{x_{1}, \ldots, x_{n}\right\}$. Geometrically, this means there is an open subscheme

$$
\operatorname{Spec}\left(\mathrm{k}\left[x_{1}^{ \pm 1}, \ldots, x_{n}^{ \pm 1}\right]\right) \subseteq \operatorname{Spec}(\mathcal{U}) .
$$

Let us call subschemes of this form cluster tori. Let $X \subseteq \operatorname{Spec}(\mathcal{U})$ be the union of the cluster tori, as $(\mathrm{Q}, \boldsymbol{x})$ runs over all seeds.

While the scheme $\operatorname{Spec}(\mathcal{U})$ is generally neither smooth nor locally finite type over k, the open subscheme $X$ is both. Hence, by Theorem A.5, we have isomorphisms

$$
f^{!}\left(\mathcal{O}_{X}\right) \stackrel{\sim}{\longrightarrow} \mathcal{H o m}_{X}\left(f^{*} \omega_{X / \mathrm{k}}, \omega_{X / \mathrm{k}}\right) \stackrel{\sim}{\longrightarrow}\left(\omega_{X / \mathrm{k}}\right)^{1-p} .
$$

Proposition A.6. Let $X$ be the union of the cluster tori in $\operatorname{Spec}(\mathcal{U})$.

(1) $\mathcal{O}_{X}(X)$ is isomorphic to $\mathcal{U}$ as a k-algebra.

(2) $f^{!}\left(\mathcal{O}_{X}\right)(X)$ is isomorphic to $\operatorname{Hom}_{\mathcal{U}}\left(\mathcal{U}, \mathcal{U}^{p}\right)$ as a $\mathcal{U}$-module.

(3) $\omega_{X / \mathrm{k}}(X)$ is isomorphic to $\omega_{\mathcal{U} / \mathrm{k}}$ as a $\mathcal{U}$-module.

On any cluster torus, these isomorphisms restrict to the obvious isomorphisms.

Proof. The first isomorphism is a standard fact about upper cluster algebras; see, e.g., [Matherne and Muller 2014, Proposition 3.4]. The other two isomorphisms follow from Theorems 3.8 and A.3, which show that $\operatorname{Hom}_{\mathcal{U}^{p}}\left(\mathcal{U}, \mathcal{U}^{p}\right)$ and $\omega_{\mathcal{U} / \mathrm{k}}$ are each free of rank one over $\mathcal{U}$ with a distinguished generator (the cluster splitting and either log volume form, respectively). On each cluster torus, the sheaves $f^{!}\left(\mathcal{O}_{X}\right)$ and $\omega_{X / \mathrm{k}}$ are free and generated by the restriction of the generator. Hence, a global section of $f^{!}\left(\mathcal{O}_{X}\right)$ or $\omega_{X / \mathrm{k}}$ can be written as the distinguished generator times a rational function which is Laurent in each cluster, that is, an element of $\mathcal{U}$.

As a consequence, we have an isomorphism of $\mathcal{U}$-modules

$$
\operatorname{Hom}_{\mathcal{U}^{p}}\left(\mathcal{U}, \mathcal{U}^{p}\right) \stackrel{\sim}{\longrightarrow}\left(\omega_{\mathcal{U} / \mathrm{k}}\right)^{1-p},
$$

where $\omega_{\mathcal{U} / \mathrm{k}}$ to a negative power means $\omega_{\mathcal{U} / \mathrm{k}}^{*}=\left(\Lambda_{\mathcal{U}}^{n} \Omega_{\mathcal{U} / \mathrm{k}}\right)^{*}$ to a positive power.

The connection between cluster splittings and log volume forms starts to become clear. Theorem A.3 establishes that $\omega_{\mathcal{U} / \mathrm{k}}$ is free of rank one as a $\mathcal{U}$-module. Hence, $\operatorname{Hom}_{\mathcal{U}}\left(\mathcal{U}, \mathcal{U}^{p}\right)$ is free of rank one as a $\mathcal{U}$-module, or equivalently, $\operatorname{Hom}_{\mathcal{U}}\left(\mathcal{U}^{1 / p}, \mathcal{U}\right)$ is free of rank one as a $\mathcal{U}^{1 / p}$-module.

To choose a distinguished generator, we observe that $\omega_{\mathcal{U} / \mathrm{k}}$ has two natural generators (the log volume forms) which differ by a sign. Since $p$ is odd, the $(1-p)$-th power of the two log volume forms coincide, so $\left(\omega_{\mathcal{U} / \mathrm{k}}\right)^{1-p}$ has a canonical generator. This determines a canonical generator in $\operatorname{Hom}_{\mathcal{U}}\left(\mathcal{U}^{1 / p}, \mathcal{U}\right)$ over $\mathcal{U}^{1 / p}$; all that remains is to observe that it coincides with the cluster splitting. 
Proposition A.7. If $\mu \in \omega_{\mathcal{U} / \mathrm{k}}$ is either log volume form, then the image of $\mu^{1-p}$ under the map

$$
\left(\omega_{\mathcal{U} / \mathrm{k}}\right)^{1-p} \stackrel{\sim}{\longrightarrow} \operatorname{Hom}_{\mathcal{U}}\left(\mathcal{U}^{1 / p}, \mathcal{U}\right)
$$

is the cluster spitting $\phi: \mathcal{U}^{1 / p} \rightarrow \mathcal{U}$.

The reader is cautioned that, as written, this is not a module map; rather, it intertwines the $\mathcal{U}$-action on $\left(\omega_{\mathcal{U} / \mathrm{k}}\right)^{1-p}$ and the $\mathcal{U}^{1 / p}$-action on $\operatorname{Hom}_{\mathcal{U}}\left(\mathcal{U}^{1 / p}, \mathcal{U}\right)$.

Proof. If $L_{\boldsymbol{x}}$ is the k-Laurent ring in some cluster $\boldsymbol{x}$, the localization map $\mathcal{U} \subset L_{\boldsymbol{x}}$ induces localization maps

$$
\left(\omega_{\mathcal{U} / \mathrm{k}}\right)^{1-p} \hookrightarrow\left(\omega_{L_{\boldsymbol{x}} / \mathrm{k}}\right)^{1-p} \quad \text { and } \quad \operatorname{Hom}_{\mathcal{U}}\left(\mathcal{U}^{1 / p}, \mathcal{U}\right) \hookrightarrow \operatorname{Hom}_{L_{\boldsymbol{x}}}\left(L_{\boldsymbol{x}}^{1 / p}, L_{\boldsymbol{x}}\right)
$$

It suffices to check that $\mu^{1-p}$ is sent to the standard splitting of $L_{\boldsymbol{x}}$; this is essentially [Brion and Kumar 2005, Lemma 1.3.6].

\section{Acknowledgements}

We are grateful to MSRI for financial support and for organizing the workshop in August 2012 designed to foster interactions between commutative algebra and cluster algebras, where this collaboration was born.

We are also indebted to Allen Knutson and David Speyer, who graciously shared their unpublished work on Frobenius splittings of cluster algebras.

\section{References}

[Berenstein et al. 2005] A. Berenstein, S. Fomin, and A. Zelevinsky, "Cluster algebras III: upper bounds and double Bruhat cells", Duke Math. J. 126:1 (2005), 1-52. MR 2005i:16065 Zbl 1135.16013

[Brion and Kumar 2005] M. Brion and S. Kumar, Frobenius splitting methods in geometry and representation theory, Progress in Mathematics 231, Birkhäuser, Boston, 2005. MR 2005k:14104 Zbl 1072.14066

[Bruns and Herzog 1993] W. Bruns and J. Herzog, Cohen-Macaulay rings, Cambridge Studies in Advanced Mathematics 39, Cambridge University Press, 1993. MR 95h:13020 Zbl 0788.13005

[Elkik 1981] R. Elkik, "Rationalité des singularités canoniques", Invent. Math. 64:1 (1981), 1-6. MR 83a:14003 Zbl 0498.14002

[Fock and Goncharov 2007] V. V. Fock and A. B. Goncharov, "Dual Teichmüller and lamination spaces", pp. 647-684 in Handbook of Teichmüller theory. Vol. I, edited by A. Papadopoulos, IRMA Lect. Math. Theor. Phys. 11, Eur. Math. Soc., Zürich, 2007. MR 2008k:32033 Zbl 1162.32009

[Fomin and Zelevinsky 2002] S. Fomin and A. Zelevinsky, "Cluster algebras I: foundations", J. Amer. Math. Soc. 15:2 (2002), 497-529. MR 2003f:16050 Zbl 1021.16017

[Fomin and Zelevinsky 2003] S. Fomin and A. Zelevinsky, " $Y$-systems and generalized associahedra", Ann. of Math. (2) 158:3 (2003), 977-1018. MR 2004m:17010 Zbl 1057.52003

[Gekhtman et al. 2005] M. Gekhtman, M. Shapiro, and A. Vainshtein, "Cluster algebras and WeilPetersson forms”, Duke Math. J. 127:2 (2005), 291-311. MR 2006d:53103 Zbl 1079.53124 
[Hara and Watanabe 2002] N. Hara and K.-I. Watanabe, "F-regular and F-pure rings vs. log terminal and log canonical singularities", J. Algebraic Geom. 11:2 (2002), 363-392. MR 2002k:13009 Zbl 1013.13004

[Hartshorne 1966] R. Hartshorne, Residues and duality, Lecture Notes in Mathematics 20, Springer, Berlin-New York, 1966. MR 36 \#5145 Zbl 0212.26101

[Hochster and Huneke 1988] M. Hochster and C. Huneke, "Tightly closed ideals", Bull. Amer. Math. Soc. (N.S.) 18:1 (1988), 45-48. MR 89b:13003 Zbl 0674.13003

[Hochster and Huneke 1989] M. Hochster and C. Huneke, "Tight closure and strong $F$-regularity", pp. 119-133 in Colloque en l'honneur de Pierre Samuel (Orsay, 1987), Mém. Soc. Math. France (N.S.) 38, Société Mathématique de France, Paris, 1989. MR 91i:13025 Zbl 0699.13003

[Kontsevich and Soibelman 2013] M. Kontsevich and Y. Soibelman, "Wall-crossing structures in Donaldson-Thomas invariants, integrable systems and mirror symmetry", preprint, 2013. arXiv 1303.3253

[Kunz 1969] E. Kunz, "Characterizations of regular local rings for characteristic p", Amer. J. Math. 91 (1969), 772-784. MR 40 \#5609 Zbl 0188.33702

[Matherne and Muller 2014] J. Matherne and G. Muller, "Computing upper cluster algebras", Int. Math. Res. Not. 2014 (online publication March 2014).

[Muller 2012] G. Muller, "Skein algebras and cluster algebras of marked surfaces”, preprint, 2012. arXiv 1204.0020

[Muller 2013] G. Muller, "Locally acyclic cluster algebras”, Adv. Math. 233 (2013), 207-247. MR 2995670 Zbl 1279.13032

[Muller 2014] G. Muller, “ $\mathcal{A}=\mathcal{U}$ for locally acyclic cluster algebras”, SIGMA Symmetry Integrability Geom. Methods Appl. 10 (2014), Paper 094, 8. MR 3261850 Zbl 06359376

[Muller and Speyer 2014] G. Muller and D. E. Speyer, "Cluster algebras of Grassmannians are locally acyclic", preprint, 2014. arXiv 1401.5137

[Musiker et al. 2013] G. Musiker, R. Schiffler, and L. Williams, "Bases for cluster algebras from surfaces”, Compos. Math. 149:2 (2013), 217-263. MR 3020308 Zbl 1263.13024

[Schwede and Smith 2010] K. Schwede and K. E. Smith, "Globally $F$-regular and log Fano varieties", Adv. Math. 224:3 (2010), 863-894. MR 2011e:14076 Zbl 1193.13004

[Schwede and Tucker 2012] K. Schwede and K. Tucker, "A survey of test ideals", pp. 39-99 in Progress in commutative algebra 2, edited by C. Francisco et al., Walter de Gruyter, Berlin, 2012. MR 2932591 Zbl 1254.13007

[Shen and Goncharov 2015] L. Shen and A. B. Goncharov, "Geometry of canonical bases and mirror symmetry", Invent. Math. (online publication February 2015).

[Smith 1997a] K. E. Smith, "F-rational rings have rational singularities”, Amer. J. Math. 119:1 (1997), 159-180. MR 97k:13004 Zbl 0910.13004

[Smith 1997b] K. E. Smith, "Vanishing, singularities and effective bounds via prime characteristic local algebra”, pp. 289-325 in Algebraic geometry Santa Cruz 1995, edited by J. Kollár et al., Proc. Sympos. Pure Math. 62, Amer. Math. Soc., Providence, RI, 1997. MR 99a:14026 Zbl 0913.13004

[Smith 2000] K. E. Smith, "Globally F-regular varieties: applications to vanishing theorems for quotients of Fano varieties”, Michigan Math. J. 48 (2000), 553-572. MR 2001k:13007 Zbl 0994.14012

[Smith and Zhang 2015] K. E. Smith and W. Zhang, "Frobenius splitting in commutative algebra", in Commutative algebra and noncommutative algebraic geometry, vol. 1, Mathematical Sciences Research Institute Publications 67, Cambridge University Press, New York, 2015. 
[Speyer 2013] D. Speyer, "An infinitely generated upper cluster algebra", preprint, 2013. arXiv 1305. 6867

Communicated by Bjorn Poonen

Received 2014-09-04 Revised 2015-01-12 Accepted 2015-03-18

abenitos@umich.edu

Department of Mathematics, University of Michigan, Ann Arbor, MI 48109, United States

morilac@umich.edu

Department of Mathematics, University of Michigan, Ann Arbor, MI 48109, United States

rajchgot@umich.edu

Department of Mathematics, University of Michigan, Ann Arbor, MI 48109, United States

kesmith@umich.edu

Department of Mathematics, University of Michigan, Ann Arbor, MI 48109, United States 


\section{Algebra \& Number Theory}

msp.org/ant

\section{EDITORS}

MANAGING EDITOR

Bjorn Poonen

Massachusetts Institute of Technology

Cambridge, USA

\author{
EDITORIAL BOARD CHAIR \\ David Eisenbud \\ University of California \\ Berkeley, USA
}

\section{BOARD OF EDITORS}

Georgia Benkart

Dave Benson

Richard E. Borcherds

John H. Coates

J-L. Colliot-Thélène

Brian D. Conrad

Hélène Esnault

Hubert Flenner

Edward Frenkel

Andrew Granville

Joseph Gubeladze

Roger Heath-Brown

Craig Huneke

Kiran S. Kedlaya

János Kollár

Yuri Manin

Barry Mazur

Philippe Michel
University of Wisconsin, Madison, USA

University of Aberdeen, Scotland

University of California, Berkeley, USA

University of Cambridge, UK

CNRS, Université Paris-Sud, France

Stanford University, USA

Freie Universität Berlin, Germany

Ruhr-Universität, Germany

University of California, Berkeley, USA

Université de Montréal, Canada

San Francisco State University, USA

Oxford University, UK

University of Virginia, USA

Univ. of California, San Diego, USA

Princeton University, USA

Northwestern University, USA

Harvard University, USA

École Polytechnique Fédérale de Lausanne
Susan Montgomery

Shigefumi Mori

Raman Parimala

Jonathan Pila

Anand Pillay

Victor Reiner

Peter Sarnak

Joseph H. Silverman

Michael Singer

Vasudevan Srinivas

J. Toby Stafford

Ravi Vakil

Michel van den Bergh

Marie-France Vignéras

Kei-Ichi Watanabe

Efim Zelmanov

Shou-Wu Zhang
University of Southern California, USA

RIMS, Kyoto University, Japan

Emory University, USA

University of Oxford, UK

University of Notre Dame, USA

University of Minnesota, USA

Princeton University, USA

Brown University, USA

North Carolina State University, USA

Tata Inst. of Fund. Research, India

University of Michigan, USA

Stanford University, USA

Hasselt University, Belgium

Université Paris VII, France

Nihon University, Japan

University of California, San Diego, USA

Princeton University, USA

\section{PRODUCTION}

production@msp.org

Silvio Levy, Scientific Editor

See inside back cover or msp.org/ant for submission instructions.

The subscription price for 2015 is US $\$ 255 /$ year for the electronic version, and $\$ 440 /$ year ( $+\$ 55$, if shipping outside the US) for print and electronic. Subscriptions, requests for back issues and changes of subscribers address should be sent to MSP.

Algebra \& Number Theory (ISSN 1944-7833 electronic, 1937-0652 printed) at Mathematical Sciences Publishers, 798 Evans Hall \#3840, c/o University of California, Berkeley, CA 94720-3840 is published continuously online. Periodical rate postage paid at Berkeley, CA 94704, and additional mailing offices.

ANT peer review and production are managed by EditFLOW ${ }^{\circledR}$ from MSP.

\section{PUBLISHED BY}

- mathematical sciences publishers

nonprofit scientific publishing

http://msp.org/

(C) 2015 Mathematical Sciences Publishers 


\section{Algebra \& Number Theory}

Volume $9 \quad$ No. $4 \quad 2015$

Motivic Donaldson-Thomas invariants of small crepant resolutions

ANDREW MORRISON and KENTARO NAGAO

Étale homotopy equivalence of rational points on algebraic varieties

AMBRUS PÁL

Fermat's last theorem over some small real quadratic fields

NUNO FREITAS and SAMIR SIKSEK

Bounded negativity of self-intersection numbers of Shimura curves in Shimura surfaces MARTIN MÖLLER and DOMINGo TOLEDO

Singularities of locally acyclic cluster algebras

Angélica Benito, Greg Muller, Jenna Rajchgot and Karen E. Smith

On an analytic version of Lazard's isomorphism

GEORG TAMME

Towards local-global compatibility for Hilbert modular forms of low weight JAMES NEWTON

Horrocks correspondence on arithmetically Cohen-Macaulay varieties

981

Francesco Malaspina and A. Prabhakar RaO

The Elliott-Halberstam conjecture implies the Vinogradov least quadratic nonresidue conjecture

TERENCE TAO 